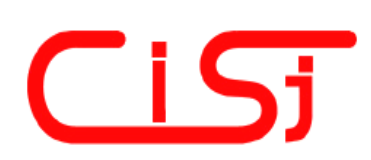

computing@tanet.edu.te.ua www.tanet.edu.te.ua/computing

\title{
A NEW HYBRID HEURISTIC TECHNIQUE FOR SOLVING JOB-SHOP SCHEDULING PROBLEM
}

\author{
Cheng-Fa Tsai, Feng-Cheng Lin \\ Department of Management Information Systems, \\ National Pingtung University of Science and Technology, Pingtung, Taiwan, 91201 \\ Email: cftsai@mail.npust.edu.tw, URL:140.127.22.200/teach/james
}

\begin{abstract}
This paper proposes a new and efficient hybrid heuristic scheme for solving job-shop scheduling problems (JSP). A new and efficient population initialization and local search concept, based on genetic algorithms, is introduced to search the solution space and to determine the global minimum solution to the JSP problem. Simulated results imply that the proposed novel JSP method (called the PLGA algorithm) outperforms several currently used approaches. This investigation also considers a real-life job-shop scheduling system design, which optimizes the performance of the job-shop scheduling system subject to a required service level. Simulation results demonstrate that the proposed method is very efficient and potentially useful in solving job-shop scheduling problems.
\end{abstract}

Keywords: JSP, genetic algorithms, local search

\section{INTRODUCTION}

Most scheduling problems are NP-hard; the time required to solve the problem optimally, increases exponentially with the size of the problem. The job-shop scheduling problem (JSP) is one of the most extensively-studied problems in combinatorial optimization. The problem may be described as follows: $n$ different jobs are to be scheduled on $m$ different machines. Each job involves a set of operations, which are performed on the machines in a prespecified order. Each operation is specified by the required machine and the fixed processing time. A number of constraints apply to the jobs and machines [26], [34]: (a) a job does not visit the same machines twice; (b) no precedence constraints are imposed among the order of different jobs; (c) an operation cannot be interrupted; (d) each machine can process only one job at a time; (e) neither release times nor due dates are specified.

The problem is to determine the order in which the jobs should be completed on the machines to minimize the makespan, which is the time required to complete all jobs. This problem has important applications, and a number of heuristic algorithms have been proposed to determine relatively good solutions to the JSP in polynomial time. They include simulated annealing (SA)[20], [28], Tabu Search [6], [17], [23], ant system (AS) [5], neural network (NN)[30], genetic algorithm (GA)[1]-[3], [4], [7]-[11], [12]-[19], [21]-[22], [24]-[27], [29]-[34], and others. Simulated annealing is used to model crystallization in thermal physics. The ant system involves a set of cooperating agents called ants, which utilize communication indirectly, by a method mediated by a pheromone. GA involves the natural selection of chromosomes. GA is known as one of the most efficient algorithms for solving the TSP (traveling salesman problem) and JSP.

\section{THE HYBRID OPTIMIZATION STRATEGY FOR JOB-SHOP SCHEDULING PROBLEM}

The GA mechanism is not governed by the utilization of differential equations, and nor does it behave like a continuous function. However, the GA mechanism does have the unique ability to identify and optimize a solution for a complex system, where other mathematical oriented techniques may fail to compile the necessary design specifications. Because of its evolutionary nature, a standard GA may not be flexible enough for practical applications, and consultation with engineers is necessary whenever a GA is applied. This becomes increasingly apparent when the problem is complicated, and involves conflict and 


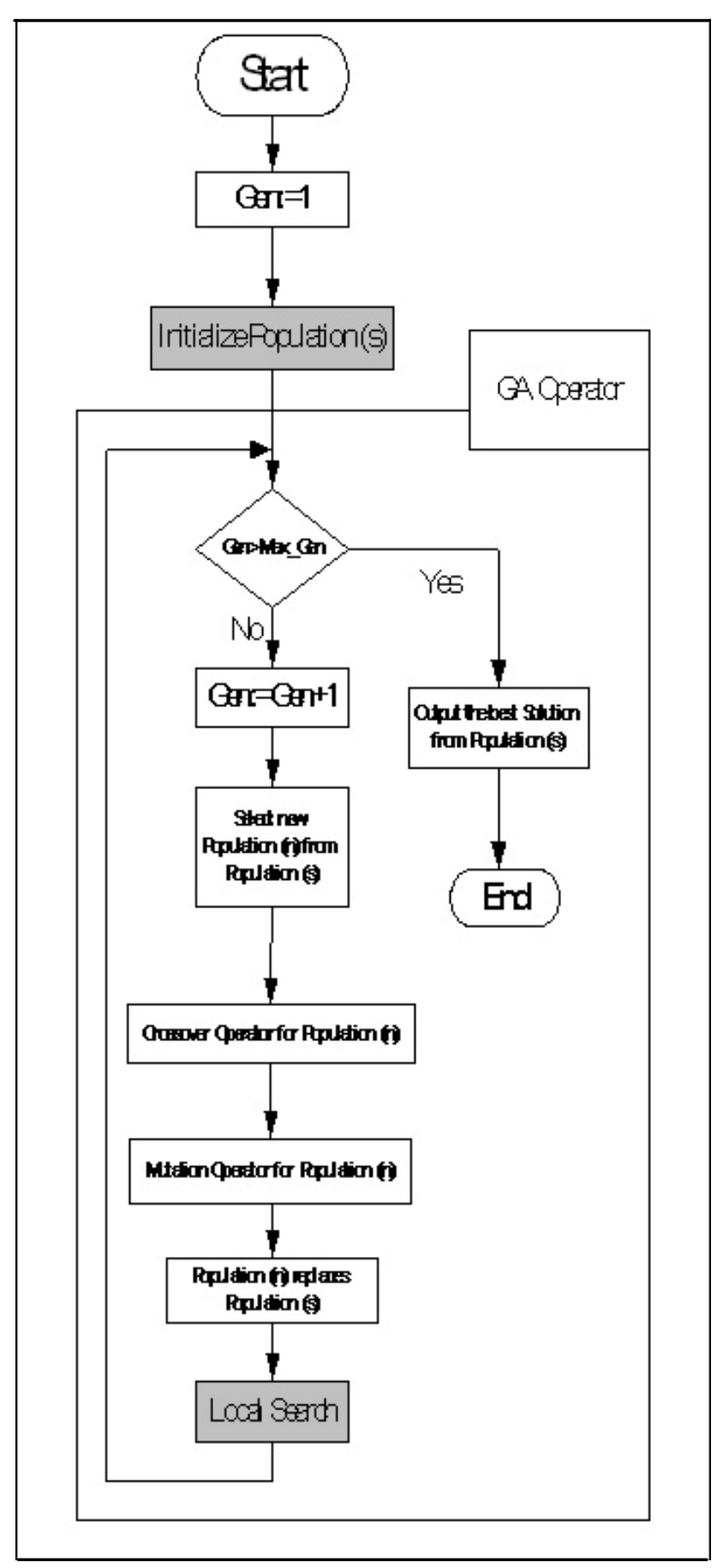

Fig. 1 - The outline of our proposed PLGA approach.

multi-tasking. Local search involves moving from a solution to another one in its neighborhood, by following many well-defined rules. The complementary strengths of GA and local search are such that a hybrid framework of GA and the local search can achieve more efficient optimization than GA alone and relax the dependence on parameters. This work presents some improvements in solving the job-shop problem obtained by taking a heuristic A method for combining other different search approaches is also provided. Fig. 1 shows the outline of our proposed PLGA approach, while Fig. 2 and 3 depict the algorithm of population initialization and local search, respectively. .

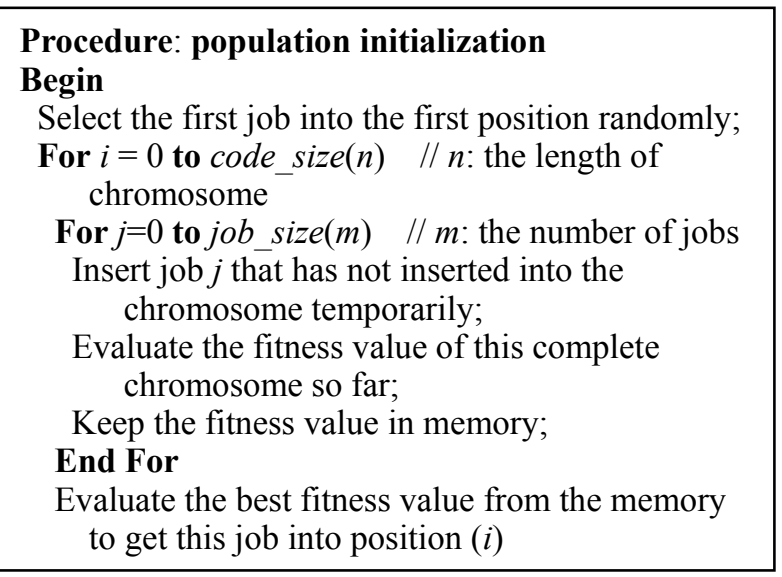

Fig. 2 - The algorithm of population initialization.

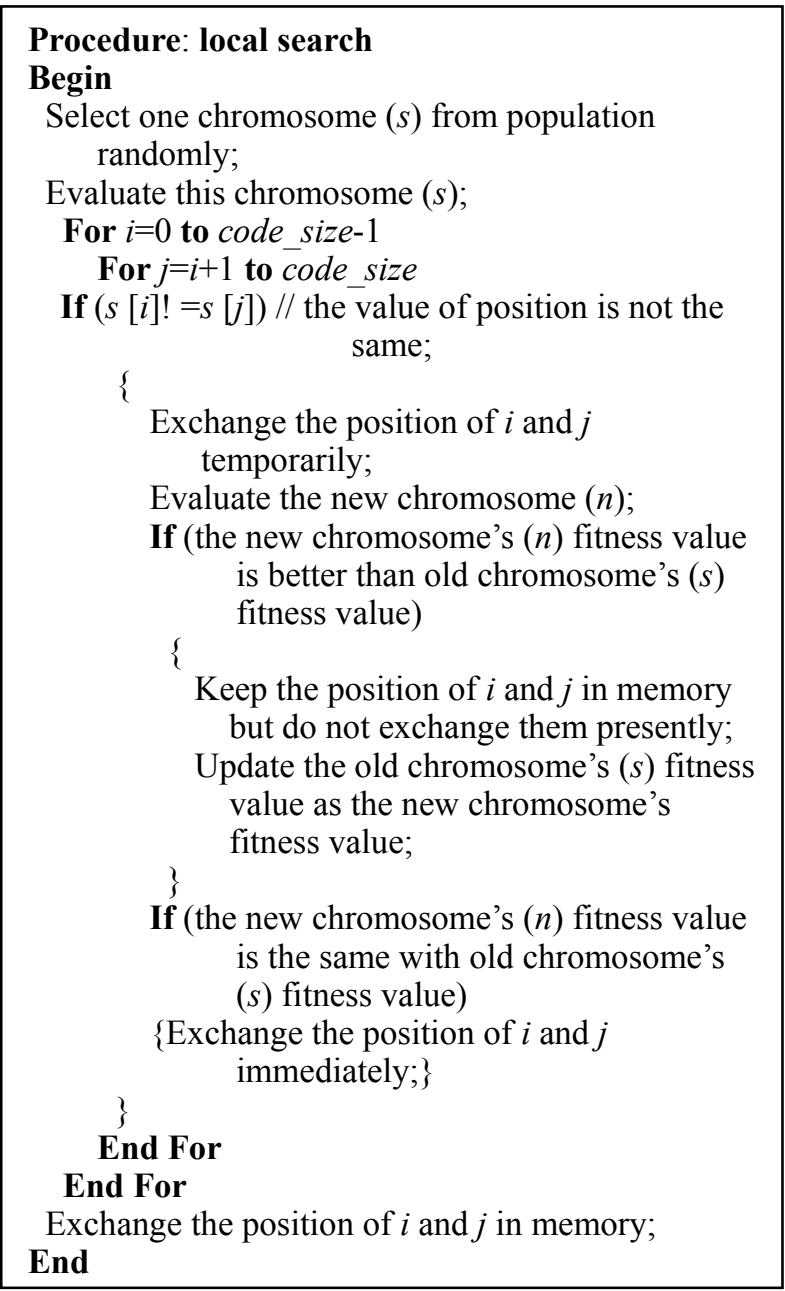

Fig. 3 - The algorithm of local search.

The mechanisms of traditional genetic algorithms are similar as illustrated in the following steps:

Step1: Initial a population of chromosomes (solutions)

Step2: Evaluate the fitness of all chromosomes 
Step3: Select fitter chromosome for reproduction

Step4: Perform crossover operator on a pair chromosome

Step5: Perform mutation operator on a chromosome

Step6: If a stopping criterion is satisfied, then stop and output the best chromosome (solution); if not, got to Step2

* Stopping criterion is mean maximum number of generations exceeded or convergence achieved.

\section{COMPUTATIONAL RESULTS}

In order to test the performances of our hybrid optimization strategy, some benchmark instances with different scale are selected and examined. These instances also are widely utilized in literatures [8], [13], [27] and are available from an anonymous ftp site ftp://mscmga.ms.ic.ac.uk/pub/jobshop1.txt. The experiments were conducted on a Pentium IV $-1.7 \mathrm{G}$ with $2 \mathrm{G}$ RAM using $\mathrm{C}++$ running on windows 2000 operating system. Meanwhile, we set Pc (crossover rate) and Pm (mutation rate) as shown in Table 2, Table 3, and Table 4, and each instance is randomly run 30 times for each algorithm. Table 1 indicates several different strategies for our proposed PLGA approach. Notably, MGA_1 uses random initialization and elitist strategy [26]; MGA_2 uses Population Initialization, elitist strategy [26]; MGA_3 uses random initialization, elitist strategy [26], and Local Search; while MGA_4 uses $50 \%$ random initialization $+50 \%$ Population Initialization, Local Search, and elitist strategy [26]. Fig. 4 reveals structure of our PLGA system, while Fig. 5 shows the viewer of our system for job-shop scheduling (an instance of optimum for 10 x10 FT). Moreover, Table 5 and Table 6

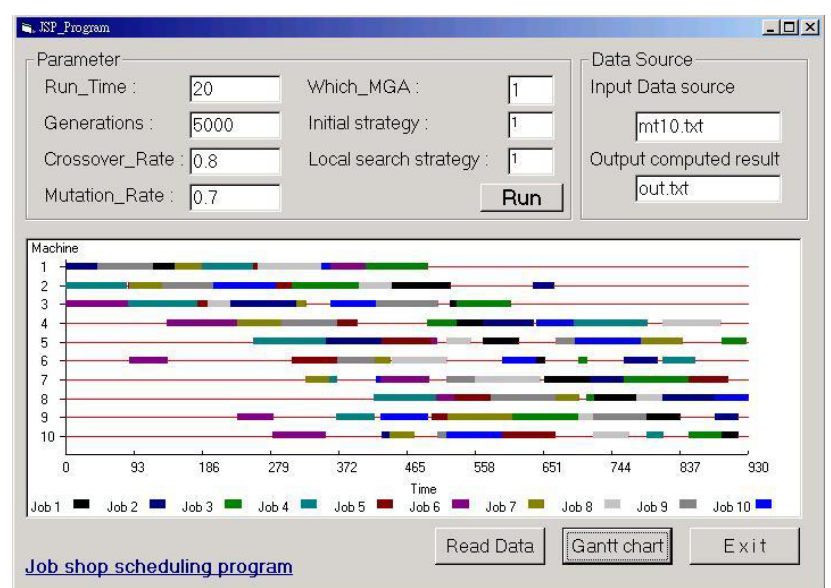

(an instance of optimum for 10 x10 FT).

Table 1. Several different strategies

\begin{tabular}{|c|c|}
\hline MGA_1 & random initialization, elitist strategy [26] \\
\hline MGA_2 & Population Initialization, elitist strategy [26] \\
\hline MGA_3 & $\begin{array}{l}\text { random initialization, elitist strategy [26], Local } \\
\text { Search }\end{array}$ \\
\hline MGA_4 & $\begin{array}{l}50 \% \text { random initialization }+50 \% \text { Population } \\
\text { Initialization, } \\
\text { Local Search, elitist strategy [26] }\end{array}$ \\
\hline
\end{tabular}

indicate simulation results of GA [33], GA+MGA_1, GA+MGA_2, GA+MGA_3, and GA+MGA_4 for 10x10FT (optimal: 930) and 20x5FT (optimal: 1165), respectively. In Table 5 and Table 6 , the proposed methods (four different strategies) can obtain better solutions than GA [33] does for 10x10FT and 20x5FT. Table 7 and Table 8 depict comparisons of the best performance between our GA (PLGA; MGA_4) and some literature results [18], [19], respectively. Such a result already demonstrated its effectiveness in finding good solutions. To the best of our knowledge and simulation results [1]-[34], our proposed approach clearly outperforms the already existing algorithms in most of job-shop scheduling problem. Due to the limitation of paper length, we cannot list all comparisons of performance with the already existing methods. In sum, the results reveal that the proposed algorithm is, indeed, a good method for solving the job-shop scheduling problem.

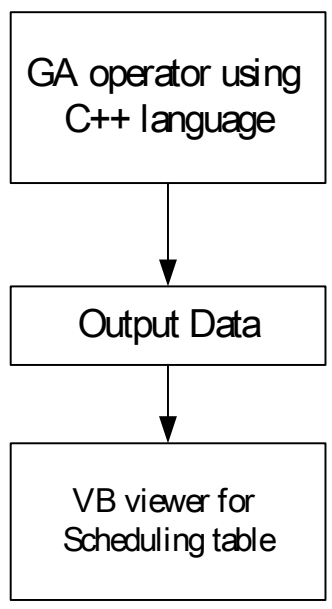

Fig. 4 - Structure of our PLGA system.

Fig. 5 - The viewer of our system for job-shop scheduling. 
Table 2. Crossover rates and mutation rates for FT benchmark problems

\begin{tabular}{|c|c|c|c|c|c|c|}
\hline \multirow{2}{*}{} & \multicolumn{3}{|c|}{ 6x6 FT \& 10x10 FT } & \multicolumn{3}{c|}{ 20x5 FT } \\
\cline { 2 - 7 } & GA & SyGA 1 & SyGA 2 & GA & SyGA 1 & SyGA 2 \\
\hline $\mathrm{Pc}$ & 0.6 & 0.8 & 0.8 & 0.6 & 1 & 0.6 \\
\hline $\mathrm{Pm}$ & 0.3 & 0.7 & 0.9 & 1 & 0.8 & 0.7 \\
\hline
\end{tabular}

Table 3. Crossover rates and mutation rates for LA benchmark problems

\begin{tabular}{|c|c|c|c|}
\hline SyGA1 & LA01. LA06. LA11. LA16. LA21 & SyGA1 & LA26. LA31. LA36. \\
\hline $\mathrm{Pc}$ & 0.8 & $\mathrm{Pc}$ & 1 \\
\hline $\mathrm{Pm}$ & 0.7 & $\mathrm{Pm}$ & 0.8 \\
\hline
\end{tabular}

Table 4. Crossover rates and mutation rates for ORB benchmark problems

\begin{tabular}{|c|c|}
\hline SyGA1 & ORB01. ORB04 \\
\hline Pc & 0.8 \\
\hline Pm & 0.7 \\
\hline
\end{tabular}

Table 5. Results for 10x10FT (optimal: 930)

\begin{tabular}{|c|c|c|c|c|c|}
\hline & GA [33] & GA +MGA_1 & GA +MGA_2 & GA +MGA_3 & GA +MGA_4 \\
\hline Best & 966 & 950 & 941 & 930 & 937 \\
\hline Average & 993 & 979.8 & 970.05 & 952.4 & 955.65 \\
\hline \multirow[t]{2}{*}{ Std div } & 19.45 & 15.6629 & 15.3742 & 10.3842 & 9.75098 \\
\hline & SyGA_1 [33] & $\begin{array}{l}\text { SYGA_1 } \\
+ \text { MGA_1 }\end{array}$ & $\begin{array}{l}\text { SyGA_1 } \\
+\mathrm{MGA} 2\end{array}$ & $\begin{array}{l}\text { SyGA_1 } \\
+\mathrm{MGA} 3\end{array}$ & $\begin{array}{c}\text { SyGA_1+ } \\
\text { MGA_4 }\end{array}$ \\
\hline Best & 937 & 937 & 945 & 935 & 930 \\
\hline Average & 965.85 & 969.3 & 961.2 & 953.5 & 954.9 \\
\hline \multirow[t]{2}{*}{ Std div } & 15.85 & 15.8748 & 6.75589 & 12.2496 & 12.6876 \\
\hline & SyGA_2 [33] & $\begin{array}{l}\text { SyGA_2 } \\
+ \text { MGA_1 }\end{array}$ & $\begin{array}{l}\text { SyGA_2 } \\
+\mathrm{MGA} 2 \\
\end{array}$ & $\begin{array}{l}\text { SyGA_2 } \\
+\mathrm{MGA} 3\end{array}$ & $\begin{array}{l}\text { SyGA_2 } \\
+ \text { MGA_4 } \\
\end{array}$ \\
\hline Best & 930 & 937 & 941 & 930 & 937 \\
\hline Average & 965.5 & 961.8 & 961.45 & 948.8 & 952.95 \\
\hline Std div & 17.09 & 14.6309 & 8.23647 & 12.9883 & 11.0047 \\
\hline
\end{tabular}

Table 6. Results for 20x5FT (optimal: 1165)

\begin{tabular}{|c|c|c|c|c|c|}
\hline & GA [33] & GA+MGA_1 & GA +MGA_2 & GA +MGA_3 & GA +MGA_4 \\
\hline Best & 1210 & 1197 & $\mathbf{1 1 7 3}$ & 1179 & 1178 \\
\hline Average & 1251.1 & 1226.45 & 1212.2 & 1191.7 & $\mathbf{1 1 9 1 . 3 5}$ \\
\hline Std div & 24.21 & 14.2366 & 16.3405 & 11.8726 & $\mathbf{8 . 8 7 5 5}$ \\
\hline & SyGA_1 [33] & SyGA_1+MGA_1 & SyGA_1+MGA_2 & SyGA_1+MGA_3 & SyGA_1+MGA_4 \\
\hline Best & 1189 & 1203 & 1176 & 1178 & $\mathbf{1 1 7 5}$ \\
\hline
\end{tabular}




\begin{tabular}{|c|c|c|c|c|c|}
\hline Average & 1214.9 & 1226.8 & 1202.8 & 1195.7 & $\mathbf{1 1 9 2 . 8}$ \\
\hline Std div & 15.51 & 16.1916 & 15.9427 & $\mathbf{1 3 . 6 5 4}$ & 13.7596 \\
\hline & SyGA_2 [33] & SyGA_2+MGA_1 & SyGA_2+ MGA_2 & SyGA_2+MGA_3 & SyGA_2+MGA_4 \\
\hline Best & 1178 & 1193 & 1177 & 1178 & $\mathbf{1 1 7 3}$ \\
\hline Average & 1233.75 & 1223.35 & 1199.6 & 1197.05 & $\mathbf{1 1 9 0 . 3}$ \\
\hline Std div & 23.24 & 21.4114 & 13.382 & $\mathbf{1 0 . 7 9 7 1}$ & 11.6533 \\
\hline
\end{tabular}

Table 7. Comparisons of the best performance between our GA (PLGA) and some literature results [18]

\begin{tabular}{|c|c|c|c|c|c|c|c|c|}
\hline Problem & Job,Machine & $\begin{array}{c}\text { Best } \\
\text { known }\end{array}$ & PLGA & $\begin{array}{c}\text { GA [18] } \\
\text { best }\end{array}$ & $\begin{array}{c}\text { SA [28] } \\
\text { best }\end{array}$ & $\begin{array}{c}\text { TS [6] } \\
\text { best }\end{array}$ & $\begin{array}{c}\text { GA [11] } \\
\text { best }\end{array}$ & $\begin{array}{c}\text { SB [4] } \\
\text { best }\end{array}$ \\
\hline MT06 & 6,6 & 55 & 55 & 55 & 55 & 55 & 55 & 55 \\
\hline LA01 & 10,5 & 666 & 666 & 666 & 666 & 666 & 666 & 666 \\
\hline LA06 & 15,5 & 926 & 926 & 926 & 926 & 926 & 926 & 926 \\
\hline LA11 & 20,5 & 1222 & 1222 & 1222 & 1222 & 1222 & 1222 & 1222 \\
\hline MT10 & 10,10 & 930 & 930 & 930 & 930 & 935 & 945 & 930 \\
\hline MT20 & 20,5 & 1165 & 1173 & 1165 & 1165 & 1165 & 1178 & 1178 \\
\hline LA16 & 10,10 & 945 & 945 & 945 & 956 & 945 & 979 & 978 \\
\hline LA21 & 15,10 & 1046 & 1051 & 1058 & 1063 & 1048 & 1097 & 1084 \\
\hline LA26 & 20,10 & 1218 & 1218 & 1218 & 1218 & 1218 & 1231 & 1224 \\
\hline LA31 & 30,10 & 1784 & 1784 & 1784 & 1784 & 1784 & 1784 & 1784 \\
\hline LA36 & 15,15 & 1268 & 1279 & 1292 & 1293 & 1278 & 1305 & 1305 \\
\hline
\end{tabular}

Table 8. Comparisons of the best performance between our GA (PLGA) with MGA, SA, GA from [19]

\begin{tabular}{|c|c|c|c|c|c|c|}
\hline Problem & Job, Machine & Best known & PLGA & MGA best & SA best & GA best \\
\hline MT06 & 6,6 & 55 & 55 & 55 & 55 & 55 \\
\hline MT10 & 10,10 & 930 & 930 & 930 & 939 & 997 \\
\hline MT20 & 20,5 & 1165 & 1173 & 1165 & 1227 & 1247 \\
\hline LA01 & 10,5 & 666 & 666 & 666 & 666 & 666 \\
\hline LA06 & 15,5 & 926 & 926 & 926 & 926 & 926 \\
\hline LA11 & 20,5 & 1222 & 1222 & 1222 & 1222 & 1222 \\
\hline LA16 & 10,10 & 945 & 945 & 945 & 979 & 979 \\
\hline LA21 & 15,10 & 1046 & 1051 & 1058 & 1083 & 1156 \\
\hline LA26 & 20,10 & 1218 & 1218 & 1218 & 1253 & 1328 \\
\hline LA31 & 30,10 & 1784 & 1784 & 1784 & 1784 & 1836 \\
\hline LA36 & 15,15 & 1268 & 1279 & 1291 & 1321 & 1384 \\
\hline
\end{tabular}

\section{CONCLUSIONS}

The simulation results observed in this paper show that our proposed PLGA algorithm can be applied to obtain the optimal solution or near optimal solution for the job-shop scheduling problem.

\section{REFERENCES}

[1] Cheng-Fa Tsai, Chun-Wei Tsai, and Chin-Chang Tseng. New and efficient ant-based heuristic method for solving the traveling salesman problem, Expert Systems (accepted, will appear in vol. 20 , no. 4,2003$)$.

[2] Cheng-Fa Tsai, Chun-Wei Tsai, and Ching-Chang Tseng. ACOMAC: An Efficient Method for Solving Traveling Salesman 
Problem. 2002 IEEE International Joint Conference on Neural Network (IJCNN 2002), pp. 1540-1545, Honolulu, Hawaii, USA.

[3] Cheng-Fa Tsai, Chun-Wei Tsai, and Chi-Ping Chen. A Multiple-Searching Approach to Genetic Algorithms for Solving Large Traveling Salesman Problem. 6th Intern. Conf. on Computer Science and Informatics, pp. 362-366, Durham, NC, USA.

[4] J. Adams, E. Balas, D. Zawack. The shifting bottleneck procedures for job shop scheduling. Management Science, vol. 34, pp. 391-401, 1988.

[5] A. Colorni, M. Dorigo, V. Maniezzo, M. Trubian. Ant system for Job-shop Scheduling. JORBEL - Belgian Journal of Operations Research, Statistics and Computer Science, 34(1), pp. 39-53, 1994.

[6] Dell's Amico M, M. Turbian. Applying tabu search to the job shop scheduling problem. Annual Operations Research, 40, pp. 231-252, 1993.

[7] L. Davis. Applying adaptive algorithms to epistatic domains. In Proc. of the Inter. Joint Conf. on Artificial Intelligence, pp. 162-164, 1985.

[8] D. Applegate, W. Cook. A computational study of the job-shop scheduling instance. ORSA Journal on Computing 3, pp. 149-156, 1991.

[9] E. Taillard. Benchmarks for basic scheduling problems. European Journal of operational Research, 64, pp.278-285, 1993.

[10] E. Falkenauer, S. Bouffoix. A genetic algorithm for job shop. Proc. of the 1991 IEEE international Conference on Robotics and Automation, 1991.

[11] F. Croce, R. Tadei, G. Volta. A genetic algorithm for the job shop problem. Computers \& Operations Research, pp. 15-24, 1995.

[12] Guoyong SHI, Hitoshi IIMA, and Nobuo SANNOMIYA. A new Encoding Scheme for Solving Job Shop Problems by Genetic Algorithm. Proc. of the 35th Conference on Decision and Control Kobe, Japan, pp. 4395-4400, 1996.

[13] H. Fisher and G. Thompson. Probabilistic Learning Combinations of Local Hob-Shop Scheduling Rules. In Industrial Scheduling, J. Muth and G. Thompson eds.,Prentice-Hall, pp. 1225-1251, 1963.

[14] Hong Zhou, Yuncheng Feng, Limin Han. The hybrid heuristic genetic algorithm for job shop scheduling. Computer \& Industrial Engineering, pp. 191-200, 2001.

[15] Kyung-Mi Lee and Takeshi Yamakawa. A genetic Algorithm for General Machine Scheduling Problems. 2nd International Conference on Knowledge-based Intelligent Electronic Systems, pp. 60-66, 1998.
[16] S. Kobayashi, I One, M. Yamamura. An efficient genetic algorithm for job shop scheduling problems. Proc. of the sixth International Conference on Genetic Algorithms. San Francisco, CA: Morgan Kaufmann Publishers, pp. 506-511, 1995.

[17] Koji Morikawa, Takeshi Furuhashi, Yoshiki Uchikawa. Single Populated Genetic Algorithm and its Application to Job-shop Scheduling. Proc. of Industrial Electronics, Control, Instrumentation, and Automation on Power Electronics and Motion Control, pp. 1014-1019, 1992.

[18] L. Wang, D.-Z. Zheng. An effective hybrid optimization strategy for job-shop scheduling problems. Computers \& Operations Research 28, pp. 585-596, 2001.

[19] L. Wang and D.-Z. Zheng. A Modified Genetic Algorithm for Job Shop Scheduling. The International Journal of Advanced Manfacturing Technology by Springer-Verlag, vol. 20, pp. 72-76, 2002.

[20] M. Kolonko. Some new results on simulated annealing applied to the job shop scheduling problem. European Journal of Operational Research, pp. 123-136, 1999.

[21] M. Gen and R. Cheng. Genetic Algorithms and Engineering Design. John Wiley\&Sons, New York, 1997.

[22] M. Pinedo. Scheduling: theory, algorithm, and systemd. Prentice-Hall, Englewood Cliffs, NJ, 1995.

[23] E. Nowicki, C. Smutnicki. A Fast Taboo Search Algorithm for the Job Shop Problem. Management Science, vol. 42, pp. 797-813, 1996.

[24] K. Oey, S.J. Mason. Scheduling batch processing machines in complex job shops. Conference Proceedings of the winter on Simulation, vol. 2, pp. 1200-1207, 2001.

[25] I One, M. Yamamura, S. Kobayashi. A genetic algorithms for job-based order crossover. Proc. of the Third IEEE Conference on Evolutionary Computation. Japan, pp. 547-52, 1996.

[26] R. Cheng, Mitsuo Gen and Yasuhiro Tsujimura. A tutorial Survey of job-shop scheduling problem using genetic algorithm-I. Reprentation. Computer ind. Engng vol. 30, no. 4, pp. 983-997, 1996.

[27] S. Lawrence. Resource constrained project scheduling: an experimental investigation of heuristic scheduling techniques. Graduate School of Industrial Administration, Pittsburgh, Carnegie-Mellon University, 1984.

[28] Van Laarhoven PJM, Aarts EHL, and Lenstra JK. Job shop scheduling by simulated annealing. Operations Research, 40, pp.113-125, 1992.

[29] Xiu Li, Wenhuang Liu, Shouju Ren, and Xuerui Wang. A solution of job-shop scheduling 
problems based on genetic algorithms. IEEE International Conference on Systems, Man, and Cybernetics, vol. 3, pp. 1823 -1828, 2001.

[30] Yoon-Pin Simon Foo and Yoshiyasu Takefuji. Stochastic Neural Networks for Solving job-shop scheduling part 1. Problem representation. IEEE International Conference on Neural Networks, vol. 2, pp. 275 -282, 1988.

[31] Y. Tsujimura, M. Gen and Y. Mafune. Relations between Evaluation Functions and Schedule-structures in GA-based Job-Shop Scheduling. Technical Report of IEICE in Japanese, AI99-13, pp. 17-24, 1999.

[32] Y. Tsujimura, M. Gen and R. Cheng .Improved Genetic Algorithms for Solving Job-shop Scheduling Problem. Engineering Design and Automation, vol. 3, no. 2, pp. 133-144, 1997.

[33] Y. Tsujimura, Y. Mafune and M. Gen. Effects of Symbiotic Evolution in Genetic Algorithms for Job-Shop Scheduling. Proc. of the 34th Hawaii International Conference on System Sciences, 2001.

[34] Y. Tsujimura, T. Sugimoto, Y Mafune and M. Gen. A Genetic Algorithm for Job-shop Scheduling by Means of Symbiosis Mechanism. Proc. of the 3rd Australia-Japan Joint Workshop on Intelligent and Evolutionary Systems, Canberra, Australia, pp. 228-231, 1999

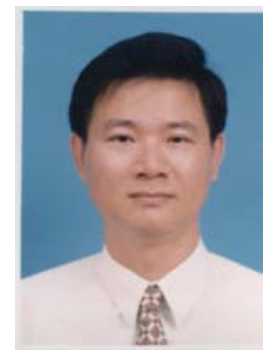

Cheng-Fa Tsai, an IEEE member, an associate professor of the Management Information Systems Department at the National Pingtung University of Science and Technology (NPUST), Pingtung, Taiwan. His research interests are in the areas of data mining, data communication networks, database systems, intelligent system, intelligent mobile agent, and wireless networks, with emphasis on efficient intelligent systems implementation and rapid prototyping. He has published over 80 papers in the above fields.

Feng-Cheng Lin received his BS degree from Ming Chuan University (Taipei, Taiwan) in 2000 and Master degree from National Pingtung University of Science and Technology (Pingtung, Taiwan) in 2002. Currently, he is a PhD student of National Chi Nan University in

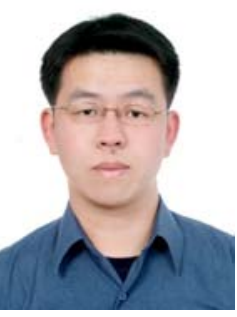
Department of Computer Science and Information Engineering (Taiwan) since 2002. His research interests are in the areas of scheduling, algorithm and the optimization of combinatorial problems. 